\title{
NEW FORMATION CONTROL DESIGNS WITH VIRTUAL LEADERS
}

\author{
Xiaorui Xi and Eyad H. Abed \\ Department of Electrical and Computer Engineering \\ and the Institute for Systems Research \\ University of Maryland, College Park, MD 20742, USA \\ $\{x x i$, abed $\} @ i s r . u m d . e d u$
}

\begin{abstract}
:
Two formation control designs are presented for flocking of a group of mobile autonomous agents in an obstacle-free environment. Both control designs use virtual leader(s) and two different interactive forces. Virtual leader(s) are used to direct the group to track a desired path, as well as to ensure the group's cohesion, via the attractive force between agents and their virtual leader(s). The repulsive force between neighboring agents is used to avoid agent collisions. It is shown that the agents can achieve a desired formation and follow the desired path at the same velocity as the virtual leader's velocity. The absence of an attractive force between neighboring agents is a new feature of this approach, meant to help reduce sensing requirements in future designs that stress a reduced level of communication among agents. Copyright (C)2005 IFAC
\end{abstract}

Keywords: nonlinear control, stability analysis, agents, sensors, target tracking

\section{INTRODUCTION}

The dynamics of flocking has in recent years received the attention of many researchers from a variety of disciplines. A flock is a group of mobile autonomous agents in which, while each agent follows certain simple rules based on local information, at the group level the agents are enabled to move together in formation and to perform desired tasks. The idea of flocking in multi-agent systems is inspired by observations in biology (Okubo, 1986; Flierl et al., 1999). Animal and insect behaviors such as swarming of ants, flocking of birds, schooling of fishes and herding of land animals are some examples of flocking in nature. Flocking can be applied in many different areas including moving in formation for fleets of unmanned aerial vehicles (UAVs), autonomous underwater vehicles (AUVs), cooperating robots, and satellite clusters.
Reynolds (1987) introduced three local rules for the motion of agents, and based on these rules developed a computer program that simulated the flocking of birds (he called the agents that obey the rules "boids." The three rules are: separation - avoiding collisions with neighboring boids; alignment - matching velocity with neighboring boids; and cohesion - staying close to the neighboring boids. Vicsek et al. (1995) proposed and simulated a simple model using only the alignment rule. Jadbabaie et al. (2003) studied this model and proved that under certain assumptions on the network connectivity, all the agents' headings converge to a common one. In the work of Olfati-Saber and Murray (2004), graph theory was used to investigate the linear consensus (alignment) problem. In the past, many researchers have made use of local attractive/repulsive potential to define the interactive force between neighboring agents to deal with 
the separation and cohesion problem (Leonard and Fiorelli, 2001; Ogren et al., 2002; Gazi and Passino, 2004; Olfati-Saber, 2004).

These three rules are only related to the achievement and maintenance of formation, i.e., to the goal of agents moving together, but which path they should follow is another issue that must be considered in flocking. Arranging that the group follows a desired path is called the tracking problem in this paper. A number of researchers have worked on the (virtual) leader/follower approach to this problem (Leonard and Fiorelli, 2001; Ogren et al., 2002; Egerstedt and Hu, 2001; Jadbabaie et al., 2003). Other researchers used the leaderless approach (Olfati-Saber, 2004).

The goal of this paper is to propose a distributed control law for a group of agents in an obstaclefree environment, whose function is to have the group track a desired path while also achieving and maintaining a desired formation.

The main contribution of this work is the introduction of two control designs for flocking based on virtual leader(s) and two different interactive forces. One is the attractive force, which is imposed on each agent by its virtual leader to achieve the goals of tracking, alignment and cohesion. The other is the repulsive force, which is imposed on each agent by its neighboring agents to solve the separation problem. The repulsive force between agents is assumed to vanish outside the immediate neighborhood of any agent. The absence of an attractive force between neighboring agents is a new feature of this approach. This may help reduce sensing requirements in future designs that stress a reduced level of communication among agents.

The remainder of the paper proceeds as follows. In Section 2, the two control designs are introduced. Analysis using LaSalle's theorem is presented in Section 3. Section 4 provides some simulation results and conclusions are given in Section 5 .

\section{PROBLEM FORMULATION}

Consider a group of $N$ identical mobile agents, modeled as point particles, moving in a plane with the following dynamics, similar to that used in (Gazi and Passino, 2004):

$$
\dot{\boldsymbol{r}}_{i}=\boldsymbol{v}_{i}
$$

where $\boldsymbol{r}_{i}, \boldsymbol{v}_{i} \in \mathbb{R}^{2}$ are the position and velocity of agent $i$. The relative displacement between agents $i$ and $j$ is denoted by $\boldsymbol{r}_{i j}=\boldsymbol{r}_{i}-\boldsymbol{r}_{j}$. The neighborhood of agent $i$ is defined as a circle of radius $d$ around agent $i$.

Suppose a desired path to the target $\boldsymbol{r}_{t}$ is given by the trajectory $\boldsymbol{p}(t)$ and $\boldsymbol{p}(t)=\boldsymbol{r}_{t}$, for $t \geq T$. The objective is to drive the agent group to track
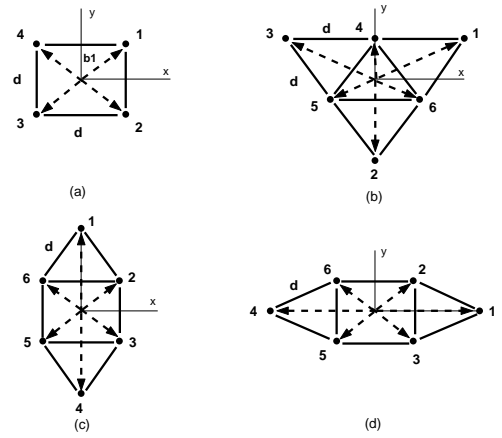

(d)

Fig. 1. Four specified desired formations

this desired path to arrive at $\boldsymbol{r}_{t}$. In this paper, two formation designs $F 1$ and $F 2$ are studied.

\subsection{F1: Specified formation ( $N$ virtual leaders)}

In this design, $N$ virtual leaders, $\boldsymbol{r}_{i d}, i=1, \ldots, N$, are introduced, one for each agent. The trajectories of these virtual leaders are given by $\boldsymbol{p}(t)+\boldsymbol{b}_{i}$, where $\boldsymbol{b}_{i} \in R^{2}, i=1, \ldots, N$, are $N$ constant vectors. The values of $\boldsymbol{b}_{i}$ s are predefined to specify the desired formation.

Fig. 1. shows four possible desired formations, where the solid dots denote the agents and any two agents linked by a solid line are neighbors. In each formation, the distance between any two neighbors is equal to $d$. The geometric center of each desired formation is taken to be the origin. So the values of $\boldsymbol{b}_{i}$ s can be calculated.

In this design, formation translation and rotation can be implemented by changing the values of $\boldsymbol{b}_{i} \mathrm{~s}$ at the instant of translation or rotation. However the change in $\boldsymbol{b}_{i}$ s needs not to be abrupt, since a relaxation period can be allowed for this change.

In this design, the values of $\boldsymbol{b}_{i}$ s must satisfy the following two conditions:(1) $\frac{1}{N} \sum_{i=1}^{N} \boldsymbol{b}_{i}=\mathbf{0}$; (2) If agent $i$ and $j$ are neighbors, then $\left\|\boldsymbol{b}_{j}-\boldsymbol{b}_{i}\right\|=d$.

In equation (1), $\boldsymbol{v}_{i}$ consists of two parts as follows

$$
\boldsymbol{v}_{i}=\boldsymbol{v}_{\mathcal{A}_{i}}+\boldsymbol{v}_{\mathcal{R}_{i}}
$$

where $\boldsymbol{v}_{\mathcal{A}_{i}}$ is the attractive component that drives agent $i$ to track its virtual leader, hence making the group to approach the desired formation, and $\boldsymbol{v}_{\mathcal{R}_{i}}$ is the repulsive part that controls the distances between agent $i$ and all its neighbors.

In $(2), \boldsymbol{v}_{\mathcal{A}_{i}}$ can be expressed in the following form:

$$
\boldsymbol{v}_{\mathcal{A}_{i}}=f\left(\left\|\boldsymbol{r}_{i d}-\boldsymbol{r}_{i}\right\|\right) \frac{\boldsymbol{r}_{i d}-\boldsymbol{r}_{i}}{\left\|\boldsymbol{r}_{i d}-\boldsymbol{r}_{i}\right\|}
$$

where $f: R_{\geq 0} \rightarrow R_{\geq 0}$ is a continuous, monotonously increasing function, which represents the magnitude of the attractive force imposed on agent $i$ by its virtual leader. The form of $f$ is illustrated in Fig. 2 (left). As can be seen, when $\left\|\boldsymbol{r}_{i d}-\boldsymbol{r}_{i}\right\|=0, f=0$, indicating that agent $i$ 


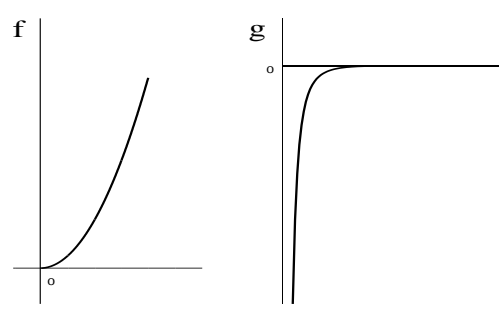

Fig. 2. The functions $f$ and $g$

has met its virtual leader at $\boldsymbol{r}_{i d}$ and no tracking. When $\left\|\boldsymbol{r}_{i d}-\boldsymbol{r}_{i}\right\|>0, f>0$, meaning that agent $i$ is being attracted toward its virtual leader.

The form of $\boldsymbol{v}_{\mathcal{R}_{i}}$ is taken to be as follows

$$
\boldsymbol{v}_{\mathcal{R}_{i}}=\sum_{j \in N_{i}(t)} g\left(\left\|\boldsymbol{r}_{j}-\boldsymbol{r}_{i}\right\|\right) \frac{\boldsymbol{r}_{j}-\boldsymbol{r}_{i}}{\left\|\boldsymbol{r}_{j}-\boldsymbol{r}_{i}\right\|}
$$

where $N_{i}(t)$ denotes the label set of agent $i$ 's neighbors at time $t$ and $g: R_{\geq 0} \rightarrow R_{\geq 0}$ is a continuous function. The function $g$ represents the magnitude of a short-range force exerted on an agent by its neighbors, whose general form is illustrated in Fig. 2 (right). As can be seen, if $\left\|\boldsymbol{r}_{j i}\right\| \geq d$, then $g=0$ and there is no g-force on agent $i$. If $0<\left\|\boldsymbol{r}_{j i}\right\|<d, g<0$, which drives agent $i$ to move away from agent $j$. Note that agent collisions are avoided by the fact that $g\left(\left\|\boldsymbol{r}_{j i}\right\|\right)$ goes to infinity as $\left\|\boldsymbol{r}_{j i}\right\|$ approaches zero.

\subsection{F2: Emergent formation (one virtual leader)}

In this design, a single virtual leader $\boldsymbol{r}_{0}$ is introduced, whose trajectory is given by $\boldsymbol{p}(t)$. Equations (1)-(4) are again employed, except that (3) is changed to be

$$
\boldsymbol{v}_{\mathcal{A}_{i}}=f\left(\left\|\boldsymbol{r}_{0}-\boldsymbol{r}_{i}\right\|\right) \frac{\boldsymbol{r}_{0}-\boldsymbol{r}_{i}}{\left\|\boldsymbol{r}_{0}-\boldsymbol{r}_{i}\right\|}
$$

Equation (5) implies that there is no a prespecified desired formation, rather all agents tend to squeeze toward the position $\boldsymbol{r}_{0}$ under the influence of the $\boldsymbol{v}_{\mathcal{A}_{i}}$ s. However, the distances between neighbors are controlled through the $\boldsymbol{v}_{\mathcal{R}_{i}}$ s so that a balanced (emergent) formation will result.

It should be noted that, in both the $F 1$ and F2 designs, the interactive force between the neighboring agents is only the repulsive force. This means that each agent dose not need to attract the neighbors far away from it, which is done by each agent using the attractive forces between it and its neighbors in the previous work (Leonard and Fiorelli, 2001; Ogren et al., 2002; Gazi and Passino, 2004; Olfati-Saber, 2004). In addition, the cohesion problem can be solved indirectly in the $F 1$ and F2 designs by the attractive forces between the agents and the virtual leader(s). As a consequence, F1 and F2 designs should be more efficient than the previous designs, without losing the cohesion of the group.

\section{ANALYSIS}

\subsection{F1: Specified formation ( $N$ virtual leaders)}

From(1)-(4), the system dynamics is rewritten as

$$
\dot{\boldsymbol{r}}_{i}=f\left(\left\|\boldsymbol{r}_{i d}-\boldsymbol{r}_{i}\right\|\right) \boldsymbol{n}_{d i}+\sum_{j \in N_{i}(t)} g\left(\left\|\boldsymbol{r}_{j i}\right\|\right) \boldsymbol{n}_{j i}
$$

where $\boldsymbol{n}_{d i}=\left(\boldsymbol{r}_{i d}-\boldsymbol{r}_{i}\right) /\left\|\boldsymbol{r}_{i d}-\boldsymbol{r}_{i}\right\|$ and $\boldsymbol{n}_{j i}=$ $\boldsymbol{r}_{j i} /\left\|\boldsymbol{r}_{j i}\right\|$. Denoting $\tilde{\boldsymbol{r}}_{i}=\boldsymbol{r}_{i}-\boldsymbol{r}_{i d}$ and $\tilde{\boldsymbol{r}}_{i j}=\tilde{\boldsymbol{r}}_{i}-\tilde{\boldsymbol{r}}_{j}$, equation (6) becomes

$$
\dot{\tilde{\boldsymbol{r}}}_{i}=-f\left(\left\|\tilde{\boldsymbol{r}}_{i}\right\|\right) \tilde{\boldsymbol{n}}_{i}+\sum_{j \in N_{i}(t)} g\left(\left\|\tilde{\boldsymbol{r}}_{j i}+\boldsymbol{b}_{j i}\right\|\right) \tilde{\boldsymbol{n}}_{j i}-\dot{\boldsymbol{r}}_{i d}
$$

where $\tilde{\boldsymbol{n}}_{i}=\tilde{\boldsymbol{r}}_{i} /\left\|\tilde{\boldsymbol{r}}_{i}\right\|, \boldsymbol{b}_{j i}=\boldsymbol{b}_{j}-\boldsymbol{b}_{i}$ and $\tilde{\boldsymbol{n}}_{j i}=\left(\tilde{\boldsymbol{r}}_{j i}+\right.$ $\left.\boldsymbol{b}_{j i}\right) /\left\|\tilde{\boldsymbol{r}}_{j i}+\boldsymbol{b}_{j i}\right\|$. Two cases are considered.

Case 1: The desired path $\boldsymbol{p}(t)$ is a straight line with $\dot{\boldsymbol{p}}(t)=\boldsymbol{q}$, where $\boldsymbol{q} \in R^{2}$ is a constant vector. So $\dot{\boldsymbol{r}}_{i d}=\boldsymbol{q}, \forall i$. One equilibrium of (7) is $\tilde{\boldsymbol{r}}_{i}^{*}=\boldsymbol{a}$, $\forall i$, where $\boldsymbol{a} \in R^{2}$ solves the following equation

$$
f(\|\boldsymbol{z}\|) \frac{\boldsymbol{z}}{\|\boldsymbol{z}\|}=-\boldsymbol{q}
$$

Since $f(\cdot)$ is continuous, monotonously increasing function, $\boldsymbol{a}$ is unique. If define $\tilde{\boldsymbol{r}}(t)=\operatorname{col}\left(\tilde{\boldsymbol{r}}_{i}(t)\right)$, then this equilibrium can be rewritten as $\tilde{\boldsymbol{r}}^{*}=$ $\mathbf{1}_{N} \otimes \boldsymbol{a}$, where $\mathbf{1}_{N} \in R^{N}$ with $\left[\mathbf{1}_{N}\right]_{i}=1, \forall i$. Here $\otimes$ denotes the Kronecker product.

Proposition 3.1. Consider system (7) with $\dot{\boldsymbol{r}}_{i d}=$ $\boldsymbol{q}$ and $f(\|\cdot\|)=k\|\cdot\|$, where $k$ is a positive constant. Then every solution of this system converges asymptotically to an equilibrium of the system.

Proof. Equation (7) becomes

$$
\dot{\boldsymbol{r}}_{i}=-k\left(\tilde{\boldsymbol{r}}_{i}-\boldsymbol{a}\right)+\sum_{j \in N_{i}(t)} g\left(\left\|\tilde{\boldsymbol{r}}_{j i}+\boldsymbol{b}_{j i}\right\|\right) \tilde{\boldsymbol{n}}_{j i}
$$

Introduce a scalar Lyapunov-type function $V_{1}$ as

$$
\begin{aligned}
V_{1}(\tilde{\boldsymbol{r}}) & =\sum_{i=1}^{N}\left(\int_{0}^{\left\|\tilde{\boldsymbol{r}}_{i}-\boldsymbol{a}\right\|} k \sigma d \sigma\right. \\
& \left.+\frac{1}{2} \sum_{j \in N_{i}(t)} \int_{d}^{\left\|\tilde{\boldsymbol{r}}_{j i}+\boldsymbol{b}_{j i}\right\|} g(\sigma) d \sigma\right)
\end{aligned}
$$

The derivative of $V_{1}$ with respect to time is

$$
\begin{aligned}
\dot{V}_{1} & =\sum_{i=1}^{N}\left(\begin{array}{c}
k\left\|\tilde{\boldsymbol{r}}_{i}-\boldsymbol{a}\right\| \frac{\left(\tilde{\boldsymbol{r}}_{i}-\boldsymbol{a}\right)^{T}}{\left\|\tilde{\boldsymbol{r}}_{i}-\boldsymbol{a}\right\|} \dot{\boldsymbol{\boldsymbol { r }}}_{i} \\
+\frac{1}{2} \sum_{j \in N_{i}} g\left(\left\|\tilde{\boldsymbol{r}}_{j i}+\boldsymbol{b}_{j i}\right\|\right) \tilde{\boldsymbol{n}}_{j i}^{T}\left(\dot{\tilde{\boldsymbol{r}}}_{j i}\right)
\end{array}\right) \\
& =-\sum_{i=1}^{N}\left(\begin{array}{c}
+\sum_{j \in N_{i}} g\left(\| \tilde{\boldsymbol{r}}_{i}-\boldsymbol{a}\right) \\
=-\sum_{i=1}^{N}\left\|\dot{\tilde{\boldsymbol{r}}}_{i}\right\|^{2}
\end{array}\right)^{T} \dot{\tilde{\boldsymbol{r}}}_{i} \\
&
\end{aligned}
$$


Note that $V_{1}(\tilde{\boldsymbol{r}}) \geq 0, \dot{V}_{1}(\tilde{\boldsymbol{r}}) \leq 0$ and $\dot{V}_{1}(\tilde{\boldsymbol{r}})=0$ if and only if $\dot{\tilde{\boldsymbol{r}}}=0$. By using LaSalle's theorem, it is shown that every solution of (9) converges asymptotically to an equilibrium of (9).

Remark 3.1. It is possible that (9) has other equilibria besides $\tilde{\boldsymbol{r}}^{*}$. However from a large number of simulations, it appears that they are unstable (saddle points). Further work will be done on this issue.

Remark 3.2. Equations (10)-(11) and the conclusion of Remark 3.1 imply that the motion of the group minimizes $V_{1}(\tilde{\boldsymbol{r}})$ to its global minimizer $\tilde{\boldsymbol{r}}^{*}$.

We have not yet shown that proposition 3.1 is applicable to nonlinear $f$. However, no exceptions have been found in the simulations.

The motion of the agent group in case 1 can be described generally in three steps. Step 1: under the influence of the attractive and repulsive forces, agent $i$ moves in such a way that $\boldsymbol{r}_{i}$ converges asymptotically to $\boldsymbol{r}_{i d}(t)+\boldsymbol{a}$. Step 2: once $\boldsymbol{r}_{i}(t)=$ $\boldsymbol{r}_{i d}(t)+\boldsymbol{a}, \forall i$, then $\dot{\boldsymbol{r}}_{i}(t)=\boldsymbol{q}, \forall i$. So alignment problem is solved and the agents' formation is the desired formation. Step 3: for $t \geq T, \boldsymbol{r}_{0} \equiv \boldsymbol{r}_{t}$, $\boldsymbol{q}=\mathbf{0} \Rightarrow \boldsymbol{a}=\mathbf{0}$, so $\boldsymbol{r}_{i}(t) \rightarrow \boldsymbol{r}_{t}+\boldsymbol{b}_{i}(t)$, the agents eventually stay around the target in the desired formation.

Case 2: the desired path $\boldsymbol{p}(t)$ is a general smooth curve with $\dot{\boldsymbol{p}}(t)=\boldsymbol{q}(t)$.

Proposition 3.2. Consider the system (7) with $\dot{\boldsymbol{r}}_{i d}(t)=\boldsymbol{q}(t)$ and $f(\|\cdot\|)=k\|\cdot\|$, where $k$ is a positive constant. Let $t_{0}, t_{1}, \ldots, t_{m}$ be an increasing sequence of time, where $t_{m}=T$. Assume that in each time interval $\left[t_{j-1}, t_{j}\right], j \in\{1, \ldots, m\}$ the change of $\boldsymbol{q}(t)$ is so small that a constant vector $\boldsymbol{q}_{j}$ can replace it. Also assume that the value of $k$ and function $g$ can be chosen such that in each interval $\left[t_{j-1}, t_{j}\right], \tilde{\boldsymbol{r}} \rightarrow \tilde{\boldsymbol{r}}_{j}^{*}$ as $t \rightarrow t_{j}$, where $\tilde{\boldsymbol{r}}_{j}^{*}=\mathbf{1}_{N} \otimes \boldsymbol{a}_{j}$ and $\boldsymbol{a}_{j}$ is the solution of (8) with $\boldsymbol{q}=\boldsymbol{q}_{j}$. Then the trajectory of every solution of this system converges asymptotically to the trajectory of $\boldsymbol{a}(t)$, where $\boldsymbol{a}(t)$ is the solution of $f(\|\boldsymbol{z}\|) \frac{\boldsymbol{z}}{\|\boldsymbol{z}\|}=\boldsymbol{q}(t)$.

Proof. the proof is based on Proposition 3.1 and is ignored here.

\subsection{F2: Emergent formation (one virtual leader)}

In this design, the desired formation is called F2 formation around $\boldsymbol{r}_{0}$.

A formation is an F2 formation around $\hat{\boldsymbol{r}}$, if it satisfies the following three conditions. (D1): there is no collision between any pair of agents.

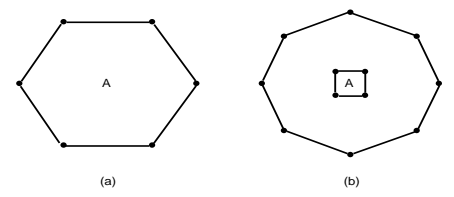

Fig. 3. (a) is a connected formation around A and (b) is a quasi-connected formation around A.

(D2): any agent has at least one neighbor, except possibly, the one at $\hat{\boldsymbol{r}}$. (D3): it is a "connected" or "quasi-connected" formation around $\hat{\boldsymbol{r}}$.

Definition 3.1. A formation is called a connected formation around $\hat{\boldsymbol{r}}=(\hat{x}, \hat{y})^{T}$, if it is a connected formation, and for any straight line defined by $\hat{y}=k \hat{x}+b$, if there is an agent $i$ at $\boldsymbol{r}_{i}$ satisfying $y_{i}>k x_{i}+b$, then there must exist at least one agent, say agent $j$ at $\boldsymbol{r}_{j}$, satisfying $y_{j}<k x_{j}+b$.

Definition 3.2. A formation is called a quasiconnected formation around $\hat{\boldsymbol{r}}$, if it contains multiple connected sub-formations, and each of them is a connected formation round $\hat{\boldsymbol{r}}$.

Fig. 3 illustrates two examples. In each plot, the solid dots denote the agents and any two agents linked by a solid line are neighbors.

From (1)-(2) and (4)-(5), the system dynamics for F2 design can be rewritten as

$$
\dot{\boldsymbol{r}}_{i}=f\left(\left\|\boldsymbol{r}_{0}-\boldsymbol{r}_{i}\right\|\right) \boldsymbol{n}_{0 i}+\sum_{j \in N_{i}(t)} g\left(\left\|\boldsymbol{r}_{j i}\right\|\right) \boldsymbol{n}_{j i}
$$

where $\boldsymbol{n}_{0 i}=\left(\boldsymbol{r}_{0}-\boldsymbol{r}_{i}\right) /\left\|\boldsymbol{r}_{0}-\boldsymbol{r}_{i}\right\|$.

Proposition 3.3. The configuration of any equilibrium of system (12) is an F2 formation around $\boldsymbol{r}_{0}$.

Proof. Any equilibrium of (12) satisfies the following condition

$$
f\left(\left\|\boldsymbol{r}_{0}-\boldsymbol{r}_{i}\right\|\right) \boldsymbol{n}_{0 i}=-\sum_{j \in N_{i}} g\left(\left\|\boldsymbol{r}_{j i}\right\|\right) \boldsymbol{n}_{j i} \neq \mathbf{0}
$$

except the agent $i$ at $\boldsymbol{r}_{i}=\boldsymbol{r}_{0}$, which satisfies

$$
f\left(\left\|\boldsymbol{r}_{0}-\boldsymbol{r}_{i}\right\|\right) \boldsymbol{n}_{0 i}=-\sum_{j \in N_{i}} g\left(\left\|\boldsymbol{r}_{j i}\right\|\right) \boldsymbol{n}_{j i}=\mathbf{0}
$$

Condition D1 is guaranteed by function $g$, and D2 is easily met because of (13) and (14).

Any equilibrium of (12) also satisfies

$$
\sum_{i=1}^{N} \dot{\boldsymbol{r}}_{i}=\sum_{i=1}^{N} f\left(\left\|\boldsymbol{r}_{0}-\boldsymbol{r}_{i}\right\|\right) \boldsymbol{n}_{0 i}=\mathbf{0}
$$

since the repulsive forces are in pairs. Define an equilibrium of (12) as $\boldsymbol{r}^{*}$. Suppose that once the group achieves the configuration of $\boldsymbol{r}^{*}$, the attractive force between the virtual leader at $\boldsymbol{r}_{0}=$ $\left(x_{0}, y_{0}\right)^{T}$ and agent $i, \forall i$, becomes a paired force, 
which means that agent $i$ also imposes a force on the virtual leader with the same magnitude as $f\left(\left\|\boldsymbol{r}_{0}-\boldsymbol{r}_{i}\right\|\right)$, but with opposite direction. By doing this, the dynamics of the system has not been changed because of the second equality of (15). For any straight line given by $y_{0}=k x_{0}+b$, if there is an agent imposing a force $\boldsymbol{f}$ on $\boldsymbol{r}_{0}$ with the direction toward the half plane $\{(x, y) \mid y>k x+$ $b\}$, then there must be at least one other agent imposing a force on $\boldsymbol{r}_{0}$ with the direction toward the other half plane $\{(x, y) \mid y<k x+b\}$ in order to balance the force $\boldsymbol{f}$. So condition D3 is satisfied by any $\boldsymbol{r}^{*}$ and the configuration of any $\boldsymbol{r}^{*}$ is a F2 formation around $\boldsymbol{r}_{0}$.

First, we analyze the behavior of the agent group after the virtual leader has arrived at the target, i.e. the system (12) with $t \geq T$. So $\boldsymbol{r}_{0} \equiv \boldsymbol{r}_{t}$ and $\dot{\boldsymbol{r}}_{0}=\mathbf{0}$. Define a scalar Lyapunov-type function $V_{2}(\boldsymbol{r})$ as follows

$$
\begin{aligned}
V_{2}(\boldsymbol{r}) & =\sum_{i=1}^{N}\left(\int_{0}^{\left\|\boldsymbol{r}_{i}-\boldsymbol{r}_{0}\right\|} f(\sigma) d \sigma\right. \\
& \left.+\frac{1}{2} \sum_{j \in N_{i}(t)} \int_{d}^{\left\|\boldsymbol{r}_{j i}\right\|} g(\sigma) d \sigma\right)
\end{aligned}
$$

and differentiate $V_{2}(\boldsymbol{r})$ with respect to time to have

$$
\dot{V}_{2}=-\sum_{i=1}^{N}\left\|\dot{\boldsymbol{r}}_{i}\right\|^{2}
$$

Note that $V_{2}(\boldsymbol{r})>0, \dot{V}_{2}(\boldsymbol{r}) \leq 0$ for $\forall \boldsymbol{r}$ and $\dot{V}_{2}(\boldsymbol{r})=0$ if and only if $\dot{\boldsymbol{r}}=\mathbf{0}$. It is shown that the motion of the agent group minimizes $V_{2}(\boldsymbol{r})$ to one of its local minimizers, which is also an equilibrium of (12) with $t \geq T$, so after the group has achieved this equilibrium, all the agents will stay there. Following proposition 3.3, the agent group can eventually achieve an F2 formation around $\boldsymbol{r}_{t}$, since $\boldsymbol{r}_{0}=\boldsymbol{r}_{t}$.

As for the behavior of the agent group before time $T$, assume that the desired path is smooth and $T$ is long enough, by doing many simulations, it is observed that within some time, the group can achieve an F2 formation around $\boldsymbol{r}_{0}$, and then all agents move in this formation at the same velocity as that of the virtual leader, until the virtual leader reaches the target.

\section{SIMULATION RESULTS}

The two control designs developed above are applied to a group of $N$ agents. In each simulation, the initial condition of the agents is given by a set of $N$ random initial positions, (uniform distributions in the area $50 \times 50)$, and zero initial velocities. The sensing radius is $d=20$. In each
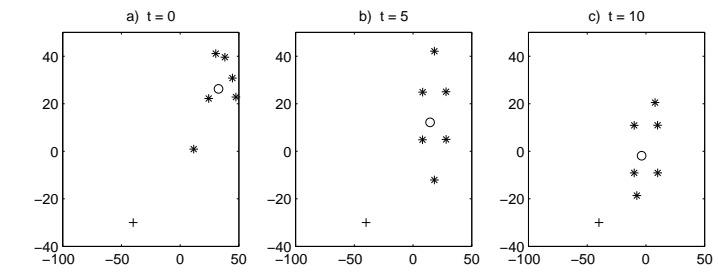

$>$
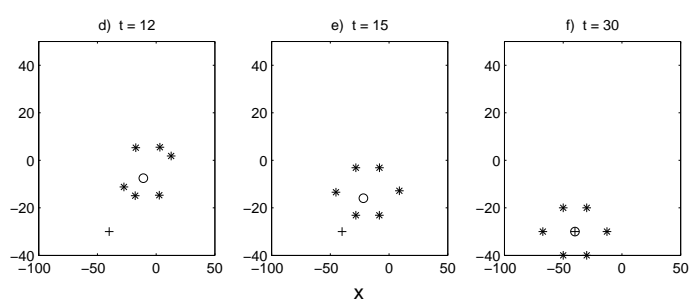

Fig. 4. 6 agents follow the virtual leader to the target and rotate from one formation to another.
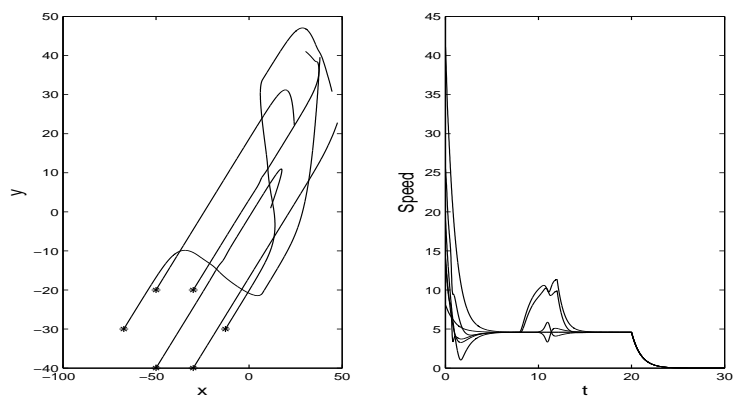

Fig. 5. the trajectories (a) and speeds (b) of the agents in $\mathrm{S} 1$

result plot, the solid dots or stars denote the agents and the cross mark denotes the target.

Simulation 1 ( $F 1$ design, Case 1): $N=6$ and $\boldsymbol{r}_{t}=(-40,-30)^{T}$. The desired path is a straight line starting from $\boldsymbol{p}(0)=\frac{\operatorname{sum}(\boldsymbol{r}(0))}{N}$. Function $f$ is linear. Predefine the values of $\boldsymbol{b}_{i}$ s such that the desired formation rotates from Fig. 1 (c) to (d) in the time interval $[8 s, 12 s]$. Fig. 4 shows the results, and in each plot, the circle denotes the position of $\boldsymbol{r}_{0}$ at the instant of that plot. Fig. 4 (a) shows the initial positions of the agents. In (b), the group has already achieved the desired formation as depicted in Fig. 1 (c). In Fig. 4 (c) and (d), the group is rotating. The group is moving toward the target in the new desired formation in (e). Finally, in (f), the formation is the desired formation and the geometric center of the group arrived at the target. Fig. 5 shows the trajectories and speeds of these agents. It can be seen that after about $5 \mathrm{~s}$, all the speeds converge to a constant, which equals to the virtual leader's speed, except for those during their rotation. After the virtual leader stop at the target, i.e. $t \geq 20 \mathrm{~s}$, their speeds decrease to zero.

Simulation 2 ( $F 1$ design, Case 2 with $f(\|\boldsymbol{z}\|)=$ $\left.0.02\left(\|\boldsymbol{z}\|^{2}+\|\boldsymbol{z}\|\right)\right): N=6$. Only look at the behavior of the group before the virtual leader stop. The total flocking time is $50 \mathrm{~s}$. Fig. 6 shows 


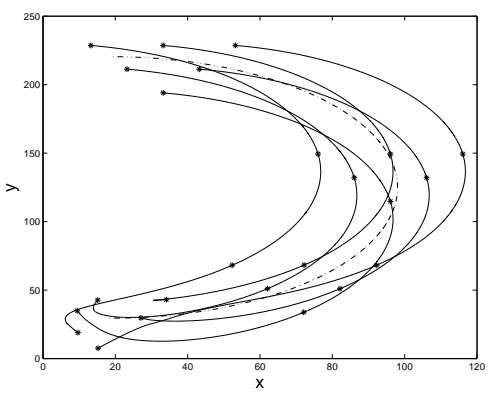

Fig. 6. The trajectories of the 6 agents, the desired path (dash-dot line) and four snaps of the agents' positions at $t=0, t=15, t=30$ and $t=50$, in the second simulation.

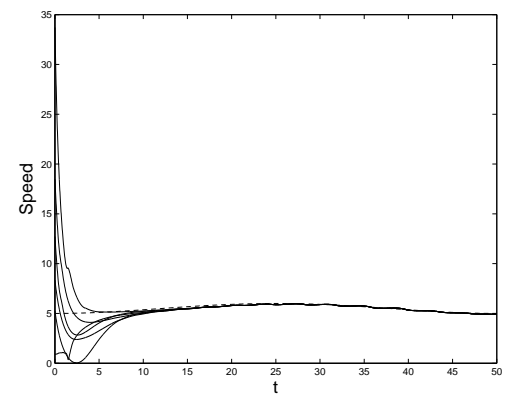

Fig. 7. The speeds of the 6 agents and the virtual leader (dash-dot line) int the second simulation.
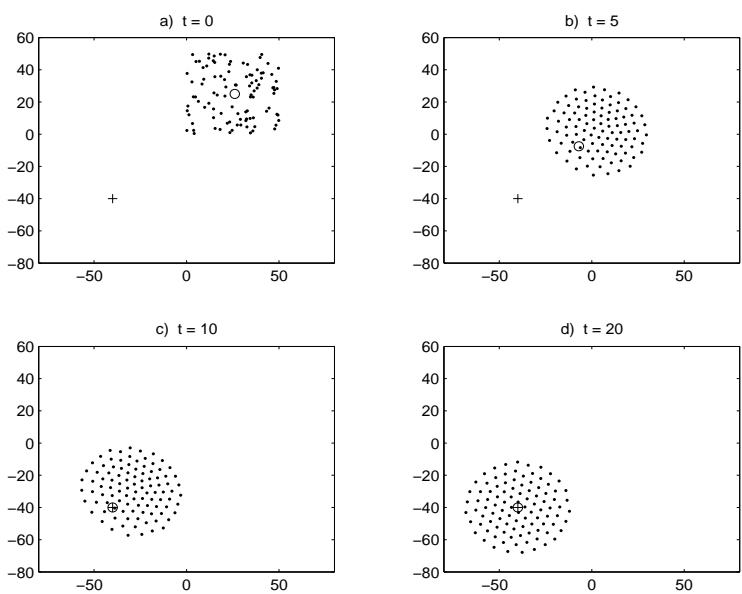

Fig. 8. The flocking of a group of 100 agents

the trajectories of all the agents, the desired path (dash-dot line), and four snaps of the agents' positions at different times. Fig. 7 shows the speeds of all the agents and the speed of the virtual leaders (dash-dot line) along time $t$.

Simulation 3 (F2 design): $N=100$ and $\boldsymbol{r}_{t}=$ $(-40,-40)^{T}$. The results are shown in Fig. 8 . It can be seen that although the agents start out completely random, they approach an F2 formation around the virtual leader after some time, whose configuration depends on the initial condition and the functions $f$ and $g$. Finally, they achieve another F2 formation around the target.

\section{CONCLUSION}

In this work, formation control laws are designed for the flocking of a group of mobile autonomous agents in an obstacle-free environment. Based on the three rules introduced by Reynolds (1987), it is concluded that there are four problems that the flocking design should solve. These are the separation, cohesion, alignment and tracking problems. In the two designs of this paper, the separation problem is solved by using the repulsive forces between any neighboring agents, and the other three problems are solved by using virtual leader(s) and attractive forces between the agents and their virtual leader(s).

\section{ACKNOWLEDGMENTS}

This work was supported in part by the U.S. Army Research Office. The authors also thank Drs. M.A. Hassouneh and D.-C. Liaw for helpful discussions.

\section{REFERENCES}

Egerstedt, M. and X. Hu (2001). Formation constrained multi-agent control. IEEE Transactions on Robotics and Automation $\mathbf{1 7}(6), 947-951$.

Flierl, G., D. Grunbaum, S. Levin and D. Olson (1999). From individuals to aggregations: the interplay between behavior and physics. Journal of Theoretical Biology 196(4), 397-454.

Gazi, V. and K.M. Passino (2004). Stability analysis of social foraging swarms. IEEE Transactions on Systems, Man, and Cybernetics-Part B: Cybernetics 34(1), 539-557.

Jadbabaie, A., J. Lin and A. S. Morse (2003). Coordination of groups of mobile autonomous agents using nearest neighbor rules. IEEE Trans. on Automatic Control 48(6), 9881001.

Leonard, N. and E. Fiorelli (2001). Virtual leaders, artificial potentials and coordinated control of groups. In: Proc. of the 40th IEEE Conference on Decision and Contro. Orlando, Florida. pp. 2968-2973.

Ogren, P., E. Fiorelli and N. Leonard (2002). Formations with a mission: stable coordination of vehicle group maneuvers. In: Proc. 15th International Symposium on Mathematical Theory of Networks and Systems. Indiana.

Okubo, A. (1986). Dynamical aspects of animal grouping: swarms, schools, flocks, and herds. Advances in Biophysics 22, 1-94.

Olfati-Saber, R. (2004). Flocking for multi-agent dynamic systems: algorithms and theory. Technical Report CIT-CDS 2004-2005. California Institute of Technology. 